\title{
Comparisons between the Wake of a Wind Turbine Generator Operated at Optimal Tip Speed Ratio and the Wake of a Stationary Disk
}

\author{
Takanori Uchida, Yuji Ohya, and Kenichiro Sugitani \\ Research Institute for Applied Mechanics, Kyushu University, 6-1 Kasugakoen, Kasuga, Fukuoka 816-8580, Japan \\ Correspondence should be addressed to Takanori Uchida, takanori1211@gmail.com
}

Received 11 November 2010; Accepted 22 February 2011

Academic Editor: Guan Yeoh

Copyright (C) 2011 Takanori Uchida et al. This is an open access article distributed under the Creative Commons Attribution License, which permits unrestricted use, distribution, and reproduction in any medium, provided the original work is properly cited.

The wake of a wind turbine generator (WTG) operated at the optimal tip speed ratio is compared to the wake of a WTG with its rotor replaced by a stationary disk. Numerical simulations are conducted with a large eddy simulation (LES) model using a nonuniform staggered Cartesian grid. The results from the numerical simulations are compared to those from wind-tunnel experiments. The characteristics of the wake of the stationary disk are significantly different from those of the WTG. The velocity deficit at a downstream distance of $10 D$ ( $D$ : rotor diameter) behind the WTG is approximately 30 to $40 \%$ of the inflow velocity. In contrast, flow separation is observed immediately behind the stationary disk $(\leq 2 D)$, and the velocity deficit in the far wake $(10 D)$ of the stationary disk is smaller than that of the WTG.

\section{Introduction}

As a countermeasure against global warming, a substantial reduction in $\mathrm{CO}_{2}$ emissions has become an urgent issue. Accordingly, the effective use of wind power energy is attracting attention as a clean and environmentally friendly solution. In Japan, the number of wind power generation facilities has been rapidly increasing to achieve the goal of 300 million $\mathrm{kW}$ of wind-generated energy in 2010. These wind power generation facilities range from those with a few wind turbine generators (WTG) to large wind farms (WF) with dozens of WTGs.

Given this background, we have developed RIAMCOMPACT (Research Institute for Applied Mechanics, Kyushu University, Computational Prediction of Airflow over Complex Terrain), a nonstationary, nonlinear wind synopsis simulator that is capable of predicting the optimum sites for wind turbine construction to the pin-point level within a target area of a few $\mathrm{km}$ or less [1]. RIAM-COMPACT can also estimate the annual energy generation and the utilized capacity of a proposed WTG with observational data. To model the wind field, RIAM-COMPACT has adopted a large-eddy simulation (LES) technique.
To achieve improved accuracy of the simulation result, a continuous effort has been made in the research and development of RIAM-COMPACT. As a part of this effort, a wake model of high accuracy is currently under development to evaluate the influence of the mutual interference between WTGs. The wake model will be able to determine an appropriate separation distance between WTGs to avoid the reduction of energy generation at a wind farm as a whole due to the mutual interference between WTGs at the farm. Development of such a wake model is necessary for the effective planning of wind farms, especially in countries with limited flat areas, including Japan, in which large WTGs are constructed in high concentrations.

When multiple WTGs are installed at a site, the following empirical values are generally considered appropriate for the separation distance between two WTGs: approximately tentimes the WTG rotor diameter in the streamwise direction and three times the WTG rotor diameter in the spanwise direction. A few wind-tunnel and field experiments have been conducted to study the wake flows of WTGs $[2,3]$. However, the characteristics of the wake flow have not been sufficiently investigated. 


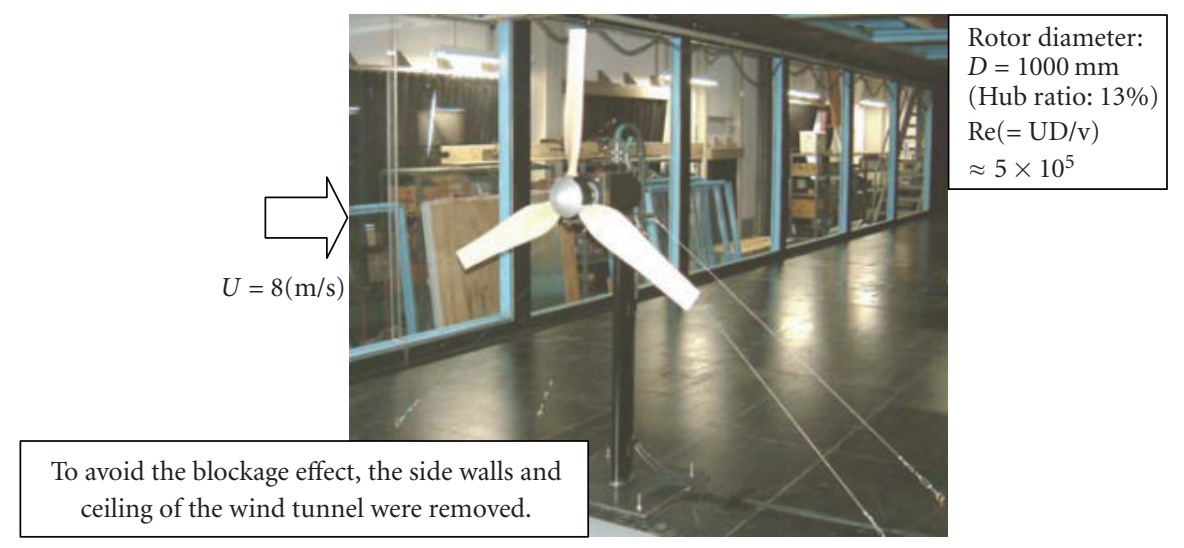

FIgURE 1: View of the small-scale WTG in the wind tunnel.

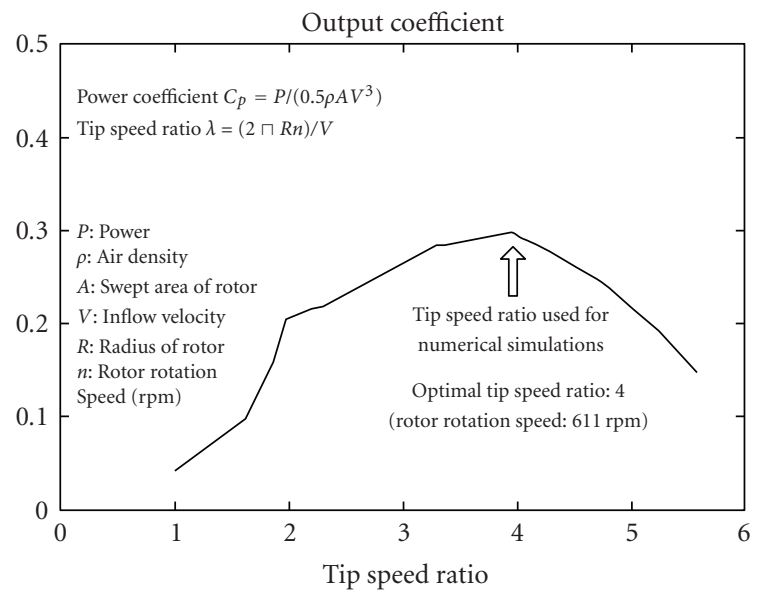

FIgURE 2: Relationship between the tip speed ratio and the power coefficient of the wind turbine generator (WTG) tested in the windtunnel experiments.

The present study examines the following two characteristics of the wake flow which will enable the construction of an accurate wake model. First, the mean wind velocity deficit is evaluated at the above-mentioned downstream distance of ten-times the rotor diameter of a single WTG. Second, the characteristics of the wake flow of a single WTG are compared to those of a stationary disk, which has been used as the basis of existing wake models. In both cases, the wake flow of the WTG operated at the optimal tip speed ratio (tip speed ratio at the maximum power output) is considered. The investigations are conducted with an LES model that uses a nonuniform staggered Cartesian grid system. The results from the numerical simulations are compared to those from wind-tunnel experiments.

\section{Numerical Simulation Technique and Results}

2.1. Numerical Simulation Technique. LES simulations are conducted using a staggered Cartesian grid with variable grid spacing. The finite difference method (FDM) is adopted for the computational technique. For the subgrid scale (SGS) model, the mixed-time scale model [4] is utilized. The mixed-time scale model is characterized by a high degree of computational stability and does not require a near-wall damping function. For explicit filtering, Simpson's rule is applied. For the pressure-velocity coupling algorithm, the fractional-step (FS) method based on the first-order explicit Euler method [5] is used. The Poisson's equation for pressure is solved by the successive overrelaxation (SOR) method. For discretization of all the spatial terms except for the convective term, a second-order central difference scheme is applied. The convective term is discretized by a third-order upwind differencing scheme, which consists of a fourth-order central differencing term based on the interpolation technique of Kajishima [6] and a numerical dispersion term in the form of the fourth derivative. A weighting value of 3.0 is normally applied as the coefficient of the numerical dispersion term in the third-order upwind differencing scheme proposed by Kawamura and Kuwahara (the Kawamura-Kuwahara Scheme) [7]. However, the coefficient is set to 0.5 in the present study to minimize the influence of numerical dispersion.

2.2. Modeling of the WTG. Figure 1 shows the small-scale WTG investigated in the wind-tunnel experiments. The blades of the WTG model are MEL airfoils [8] with increased thickness. The performance curve of the WTG determined from the wind-tunnel experiments shows that the optimal tip speed ratio of the present WTG is 4 (see arrow in Figure 2). To recreate the conditions of the wind-tunnel experiments in the numerical simulations, the configurations of the spinner, nacelle, and tower were reconstructed with a rectangular grid approximation (Figure 4). To model the rotation of the WTG rotor, an actuator-disc approach based on blade element theory $[9,10]$ is applied. In the actuator-disc approach, the tangential and thrust forces generated by the rotating blade are added to the Navier-Stokes equations as external terms. These external terms represent the reaction forces exerted on the fluid in the direction of the streamwise flow and the rotation. Thus, no wall boundary condition exists for the rotor as an object. In the present study, because a nonuniform staggered Cartesian grid system has been adopted, 


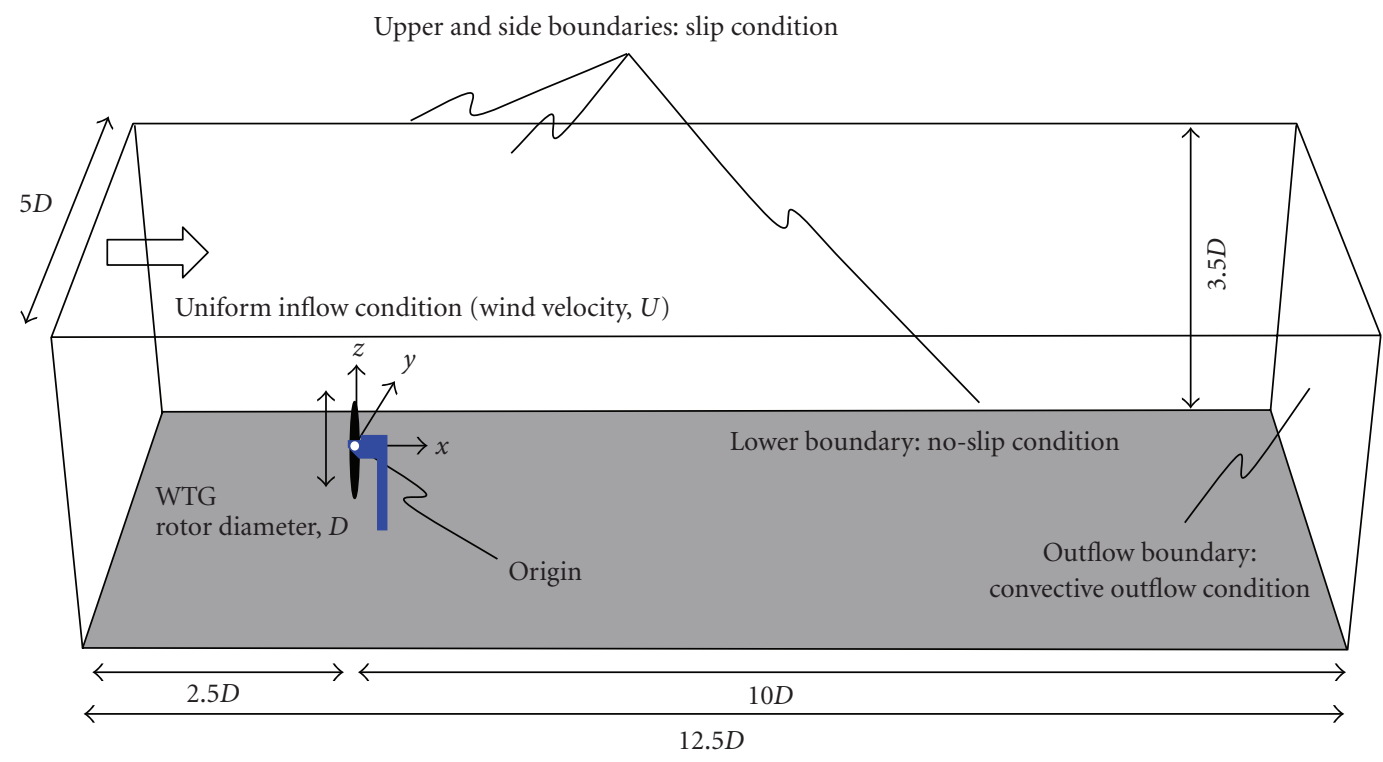

FIGURE 3: Computational domain and boundary conditions.

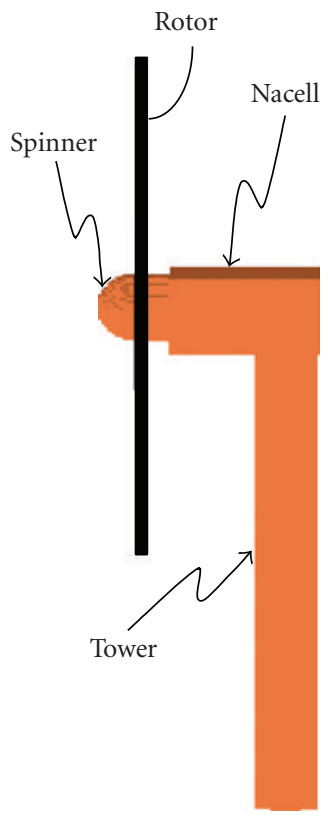

Figure 4: Enlarged view of WTG.

the component of the force in the direction of rotation is decomposed into the spanwise and vertical directions. In addition to the investigation of the decelerating effect of the WTG simply as a drag-inducing body, the adopted modeling approach allows the investigation of the effect of the blade rotation on the airflow, which is considered a major benefit of the adopted modeling approach. Furthermore, the model is designed so that it allows the user to simulate wake flows of various WTGs by inputting only the data of the blade chord length, the lift coefficient, the drag coefficient, and the angle of attack as a function of the distance from the center of the rotor. With the developed model, airflow past the entire
WTG, including the tower, is simulated for a tip speed ratio of 4 , the optimal tip speed ratio.

2.3. Computational Domain and Conditions. Numerical simulations are conducted for (1) airflow past the WTG operated at the optimal tip speed ratio and (2) airflow past a WTG for which the rotor has been replaced by a stationary disk with a diameter identical to that of the rotor. The WTG with a stationary disk will be referred to simply as the stationary disk hereafter. The computational domain and boundary conditions applied in the simulations are summarized in Figure 3. The dimensions of the computational domain are $12.5 D \times 5 D \times 3.5 D$ (streamwise $(x) \times$ spanwise $(y) \times \operatorname{vertical}(z))$, where $D$ is the rotor diameter. The computational domain consists of $181 \times 171 \times 161$ grid points (approximately 5 million grid points) in the $x, y$, and $z$ directions, respectively. Sufficiently high grid resolution is provided around the WTG to analyze the flow field past the entire WTG, including the spinner, nacelle, and tower $\left(\Delta x_{\min }=\Delta y_{\min }=\Delta z_{\min }=0.005 D\right)$. The same set of boundary conditions is applied for the two simulations except for those of the wind velocity at the rotor; for the case with the stationary disk, the wind velocity is set to zero at all grid points on the surface and in the interior of the disk. In both cases, the wind velocity is set to zero at all grid points on the surface and in the interior of the spinner, nacelle, and tower. As for the boundary conditions for pressure, Neumann boundary conditions are applied at all surfaces. The Reynolds number of the flow based on the uniform inflow wind speed, $U$, and the rotor diameter, $D$, that is, $\operatorname{Re}(=U D / v)$, is $2 \times 10^{4}$ in the present study. For simulations, a time step of $\Delta t=2 \times 10^{-3}(D / U)$ is used.

2.4. Computational Results and Discussion. Figures 5 and 6 are contour plots of the streamwise $(x)$ wind velocity, $u$, in the vicinity of the WTG operated at the optimal tip speed 


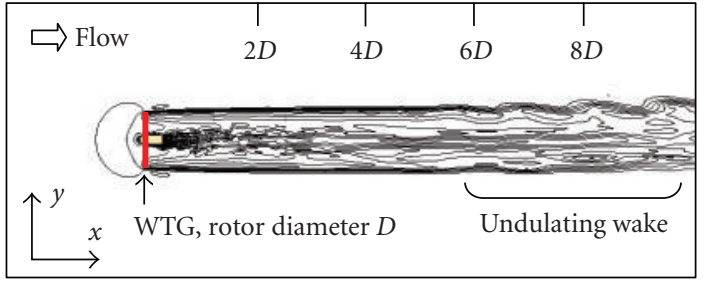

(a) WTG operated at optimal tip speed ratio

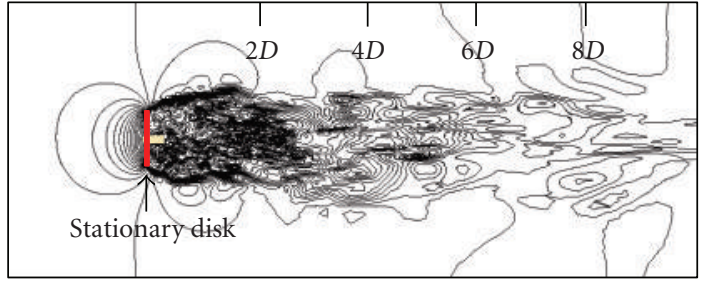

(b) Stationary disk

FigURE 5: Contour plots of the streamwise $(x)$ velocity component, $u$, with 30 equally spaced contour intervals between $u / U=-0.7$ and $u / U=1.4$, instantaneous field, top view of entire domain at $z=0$.

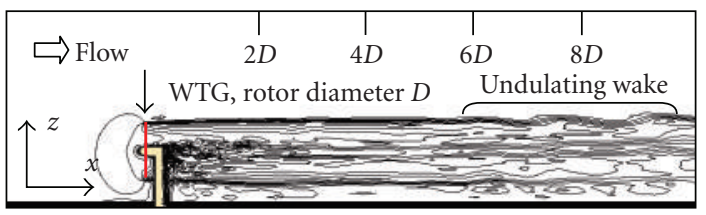

(a) WTG operated at optimal tip speed ratio

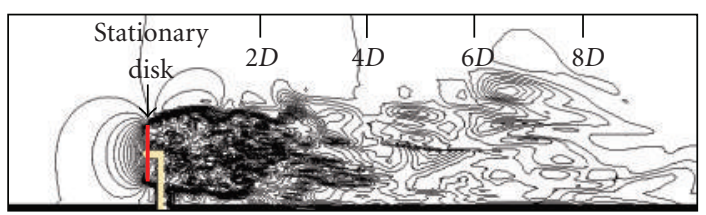

(b) Stationary disk

FIGURE 6: Contour plots of the streamwise $(x)$ velocity component, $u$, with 30 equally spaced contour intervals between $u / U=-0.7$ and $u / U=1.4$, instantaneous field, side view of entire domain at $y=0$.

ratio and in the vicinity of the stationary disk, respectively. The former figure shows a cross-section of the streamwise $(x)$ wind velocity field viewed from top at $z=0$, while the latter figure shows the same wind field viewed from the side at $y=$ 0 . In these figures, thirty equally spaced contour intervals are shown between $u / U=-0.7$ and $u / U=1.4$, and the entire computational domain is shown. The two figures suggest that the characteristics of the wake flow differ significantly between the WTG and the stationary disk. In the case of the WTG, the wake width is approximately the same as the rotor diameter, and undulating motions are observed in the wake at downstream distances larger than approximately $6 D$ as indicated in Figures 5 and 6 . Furthermore, formation of strong tip vortices is evident at the blade tips of the WTG. It can be speculated that these vortices suppress the momentum exchange between the wake and its surrounding flow, and as a result, the wake width near the WTG is reduced approximately to the rotor diameter. However, at a downstream distance of approximately $6 D$, the effect of the tip vortices becomes sufficiently weak for shear instability to cause undulations in the wake flow. In other words, the influence of the tip vortices generated at the optimal tip speed ratio extends to a downstream distance of at least $6 D$.

In contrast, the characteristics of the wake flow behind the stationary disk are complex. A large area of reverse flow is formed immediately behind the stationary disk. As a result of the flow curling around the edge of the disk, the magnitude of the spanwise component of the flow behind the stationary disk is significantly larger than that behind the WTG (not shown). From the area of reverse flow, large vortices are shed periodically. The Strouhal number of the vortex shedding and the structure of the shed vortices behind the stationary disk are topics of high interest in fluid dynamics and will be investigated in future research. Figures 7 and 8 are enlarged views of the flow near the WTG and disk in Figures 5 and 6, respectively, and also include the dynamic pressure field on the spinner, nacelle, and tower. The enlarged views provide confirmation of the above-mentioned differences between the wake flow around the WTG and that around the stationary disk. The same figures show vortex shedding from the individual components of the WTG, that is, spinner, nacelle, and tower, and the resulting formation of flow separation behind these components. Although not shown due to space limitations, a Karman vortex street was observed downstream of the WTG. These results suggest that analyses of the airflows simulated around an entire WTG rather than those around the individual components of a WTG are necessary in future discussions of vibrations of a WTG and the ability of a WTG to withstand high winds. To address these issues, the computational technique used in the present study may serve as an effective tool because it is user friendly and computationally inexpensive.

Figure 9 illustrates the streamlines of virtual fluid particles in the wake flows of the WTG operated at the optimal tip speed ratio and the stationary disk. In the case of the WTG (Figure 9(a)), a spiral flow forms as a result of the blade rotation. In contrast, in the case of the stationary disk (Figure 9(b)), complex three-dimensional streamlines are observed. These results confirm the presence of complex turbulent flow behind the stationary disk as shown in Figures 5-8.

A significant difference between the wake flow of the WTG and that of the stationary disk is also evident in the time-averaged fields of the streamwise $(x)$ component of the flow velocity (Figures 10 and 11). The time duration for obtaining the time-averaged streamwise velocity is $t=100(D / U)$. These figures show the presence of a large region of reverse flow immediately behind the stationary 


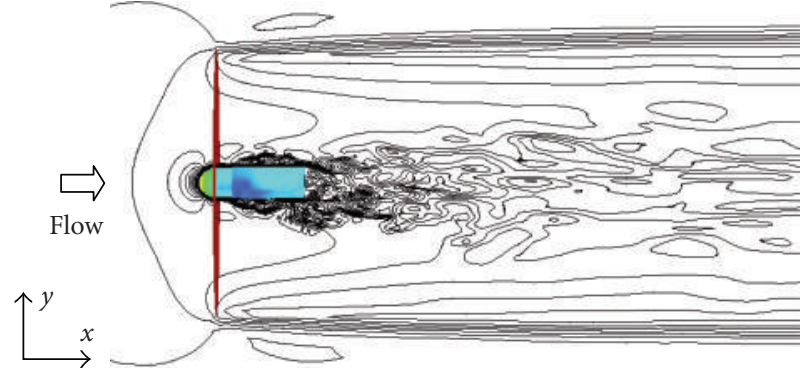

(a) WTG operated at optimal tip speed ratio

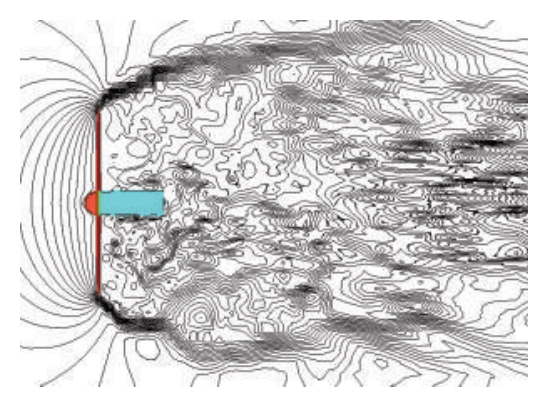

(b) Stationary disk

Figure 7: Enlarged view of Figure 5.The colors on the spinner and nacelle represent the dynamic surface pressure. Blue and red indicate the minimum (negative) and maximum values.

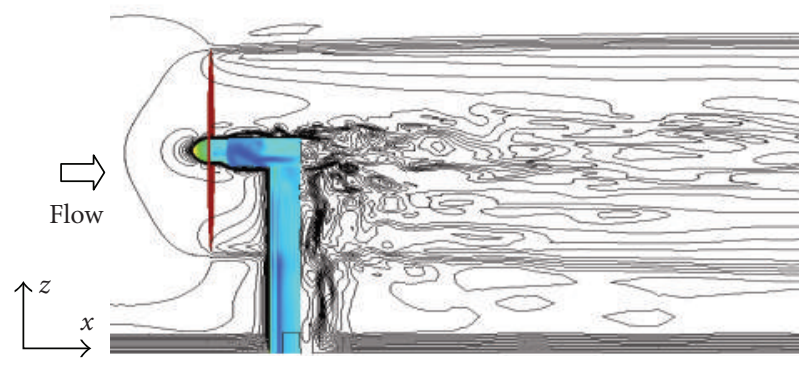

(a) WTG operated at optimal tip speed ratio

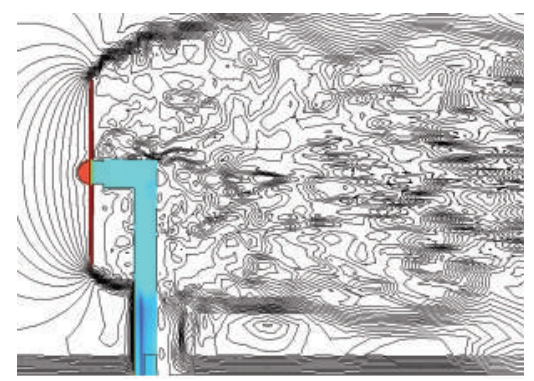

(b) Stationary disk

FIGURE 8: Enlarged view of Figure 6. The colors on the spinner, nacelle, and tower represent the dynamic surface pressure. Blue and red indicate the minimum (negative) and maximum values.

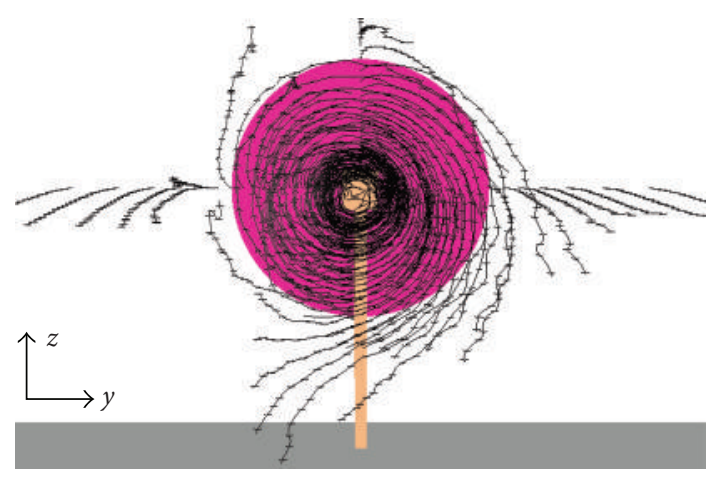

(a) WTG operated at optimal tip speed ratio

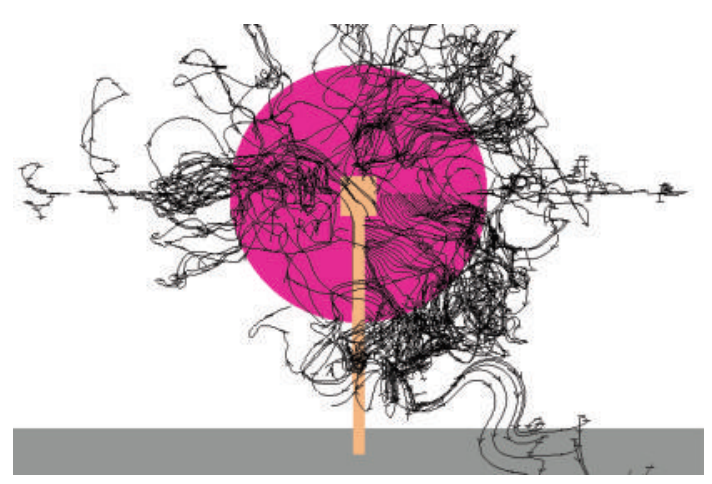

(b) Stationary disk

FIGURE 9: Flow visualization of the vortex structure of the wake flow. Rear view showing streamlines for the instantaneous field.

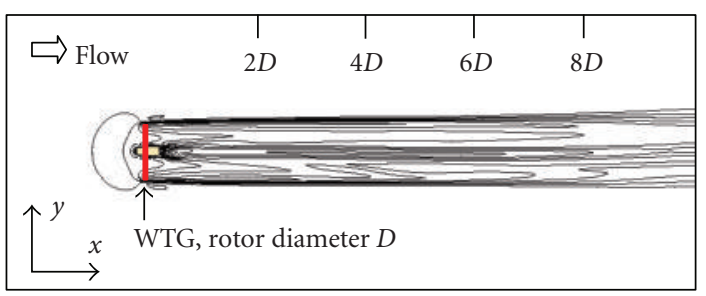

(a) WTG operated at optimal tip speed ratio

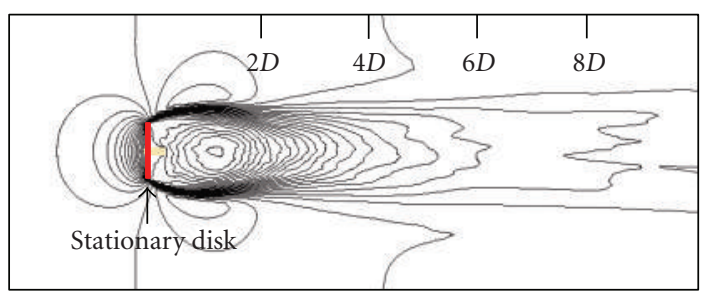

(b) Stationary disk

FIGURE 10: Contour plots of the streamwise $(x)$ velocity component, $u$, with 30 equally spaced contour intervals between $u / U=-0.7$ and $u / U=1.4$, time-averaged field, top view of the entire domain at $z=0$. 


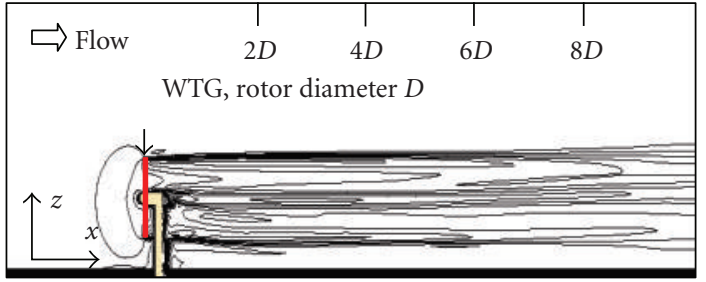

(a) WTG operated at optimal tip speed ratio

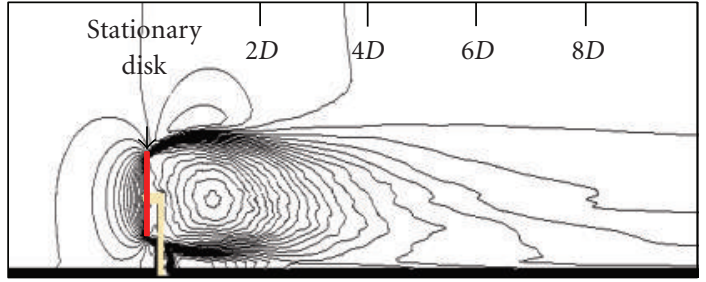

(b) Stationary disk

FIGURE 11: Same as Figure 10 except for side view of the entire domain at $y=0$.

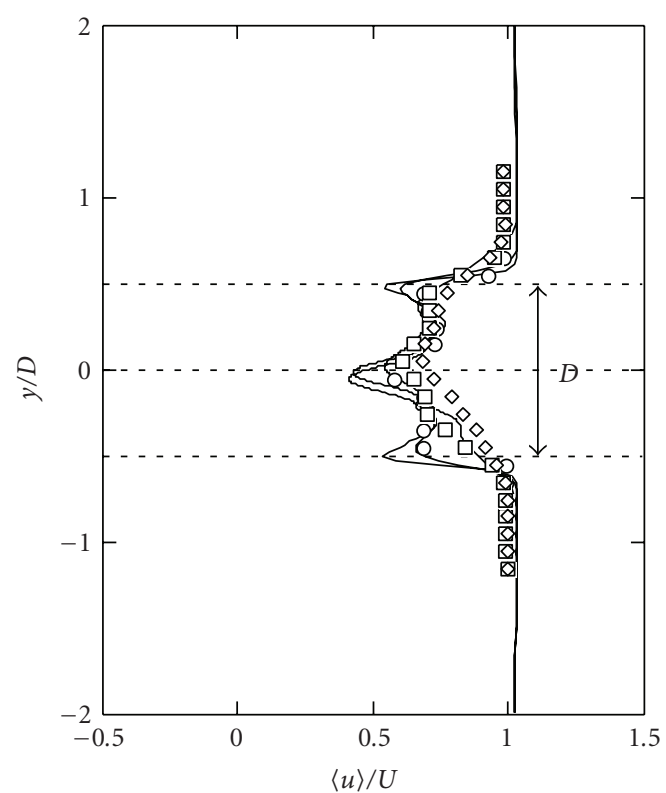

$\begin{array}{ll}\text { Wind tunnel } & \text { Simulation } \\ \circ 2 D & -\left\{\begin{array}{l}2 D \\ 6 D \\ \square\end{array} 6 D\right. \\ \diamond 10 D & -\end{array}$

(a) WTG operated at optimal tip speed ratio

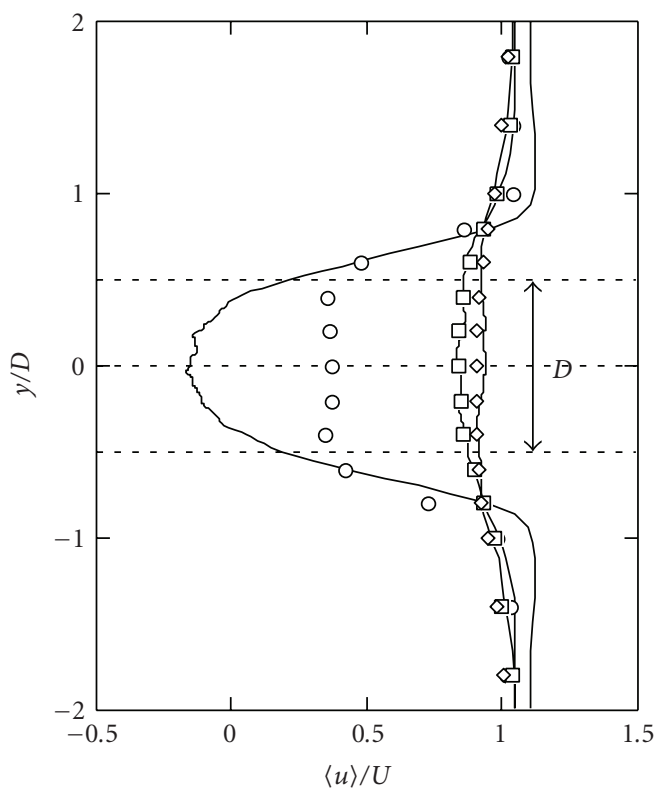

$\begin{array}{ll}\text { Wind tunnel } & \text { Simulation } \\ \circ 2 D & -\left\{\begin{array}{l}2 D \\ 6 D \\ 10 D\end{array}\right.\end{array}$

(b) Stationary disk

FIGURE 12: Spanwise profiles of the time-averaged streamwise $(x)$ velocity at $z=0((x, y, z)=(0,0,0)$ indicates the center of the rotor; see Figure 3). The profiles are observed at $x=2 D(\bigcirc), 6 D(\square)$ and $10 D(\diamond)$, where $D$ is the rotor diameter. The streamwise velocity and the spanwise position on the $y$-axis are normalized by the uniform inflow velocity, $U$, and $D$, respectively. The $y$-coordinate of the rotor is shown with arrows.

disk; however, at a downstream distance of $10 D$, the streamwise velocity deficit in the wake behind the disk is smaller than that behind the WTG.

To investigate this finding quantitatively, spanwise and vertical profiles of the time-averaged streamwise velocity are calculated (Figures 12 and 13). The profiles from the wind-tunnel experiments in these figures were obtained with an I-type hot wire probe. As for the spanwise profiles, the simulation (solid lines) and the experimental results (symbols) agree well for both the WTG and the stationary disk except for the profile at $x=2 D$ in the wake of the stationary disk. The deviation of the spanwise profile in this case is attributable to the use of an I-type probe for the airflow measurement. The streamwise velocity deficits at a downstream distance of $10 \mathrm{D}$ behind the center of the rotor are approximately $30-40 \%$ of the inflow velocity for the
WTG. These quantitative results confirm that the streamwise velocity deficit at a downstream distance of $10 D$ in the wake behind the disk is smaller than that behind the WTG. The large value of the velocity deficit behind the WTG is likely due to the tip vortices, the effect of which extends to a downstream distance of $10 D$.

\section{Conclusion}

The present study investigated the characteristics of the wake flow behind a single wind turbine generator (WTG) operated at the optimal tip speed ratio. Even at a downstream distance of as large as ten-times the rotor diameter of a single WTG, the wind velocity behind the center of the rotor was approximately $30-40 \%$ of the inflow wind velocity. This large 

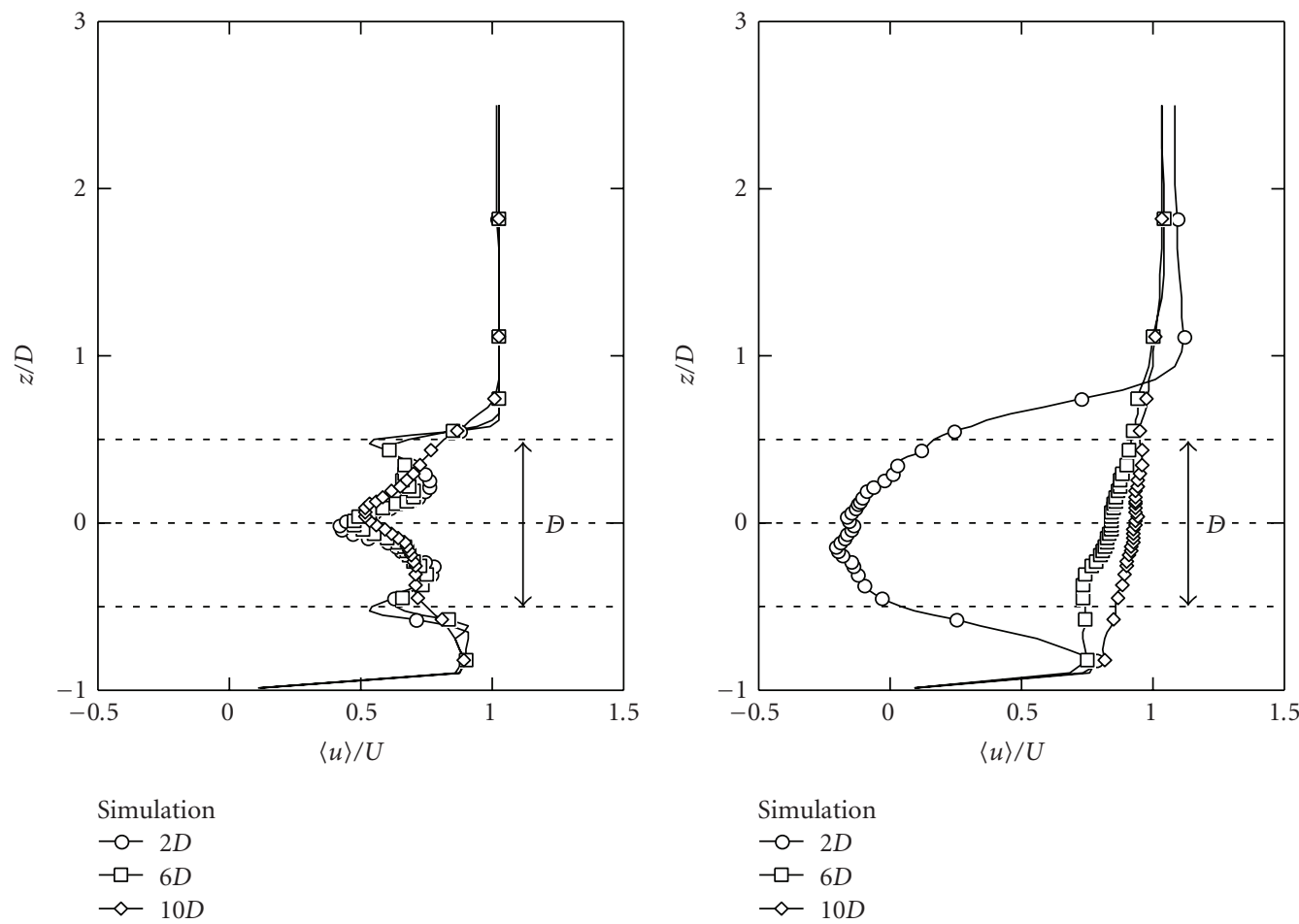

$$
\begin{aligned}
& \text { Simulation } \\
& \multimap-2 D \\
& -\square 6 D \\
& \multimap-10 D
\end{aligned}
$$

(a) WTG operated at optimal tip speed ratio

(b) Stationary disk

FIGURE 13: Same as Figure 12 except for the vertical profiles of time-averaged streamwise $(x)$ velocity at $y=0$. The $z$-coordinate of the rotor is shown with arrows.

value of the wind velocity deficit was likely attributable to the tip vortices formed at the blade tips, which suppressed the momentum exchange between the wake flow of the WTG and the surrounding flow.

The wake of the WTG was also compared to that of a WTG with the rotor replaced by a stationary disk because existing wake models are based on the wake of a stationary disk. The diameter of the stationary disk was set equal to that of the rotor of the WTG. The wake flow of the WTG and that of the stationary disk were significantly different from each other. The wake flow of the stationary disk was characterized by a large area of reverse flow immediately behind the disk. However, at a downstream distance of $10 D$, the streamwise velocity deficit in the wake behind the disk was smaller than that behind the WTG.

Our future research topics include investigations of the effects of the inflow turbulence on a WTG and turbulence distributions in the wake of a WTG. Numerical simulations of wake flows behind multiple WTGs are also planned as a future project.

\section{References}

[1] T. Uchida and Y. Ohya, "Micro-siting technique for wind turbine generators by using large-eddy simulation," Journal of Wind Engineering and Industrial Aerodynamics, vol. 96, no. 1011, pp. 2121-2138, 2008.
[2] L. P. Chamorro and F. Porté-Agel, "Effects of thermal stability and incoming boundary-layer flow characteristics on windturbine wakes: a wind-tunnel study," Boundary-Layer Meteorology, vol. 136, no. 3, pp. 515-533, 2010.

[3] Y. Käsler, S. Rahm, R. Simmet, and M. Kühn, "Wake measurements of a multi-MW wind turbine with coherent long-range pulsed doppler wind lidar," Journal of Atmospheric and Oceanic Technology, vol. 27, no. 9, pp. 1529-1532, 2010.

[4] M. Inagaki, T. Kondoh, and Y. Nagano, "A mixed-time-scale SGS model with fixed model-parameters for practical LES," Journal of Fluids Engineering, Transactions of the ASME, vol. 127, no. 1, pp. 1-13, 2005.

[5] J. Kim and P. Moin, "Application of a fractional-step method to incompressible Navier-Stokes equations," Journal of Computational Physics, vol. 59, no. 2, pp. 308-323, 1985.

[6] T. Kajishima, "Finite-difference method for convective terms using non-uniform grid," Transactions of the Japan Society of Mechanical Engineers, Part B, vol. 65, no. 633, pp. 1607-1612, 1999.

[7] T. Kawamura, H. Takami, and K. Kuwahara, "Computation of high Reynolds number flow around a circular cylinder with surface roughness," Fluid Dynamics Research, vol. 1, no. 2, pp. 145-162, 1986.

[8] http://riodb.ibase.aist.go.jp/db060/index.html.

[9] J. N. Sørensen and A. Myken, "Unsteady actuator disc model for horizontal axis wind turbines," Journal of Wind Engineering and Industrial Aerodynamics, vol. 39, no. 1-3, pp. 139-149, 1992.

[10] H. Snel, "Review of the present status of rotor aerodynamics," Wind Energy, vol. 1, pp. 46-69, 1998. 

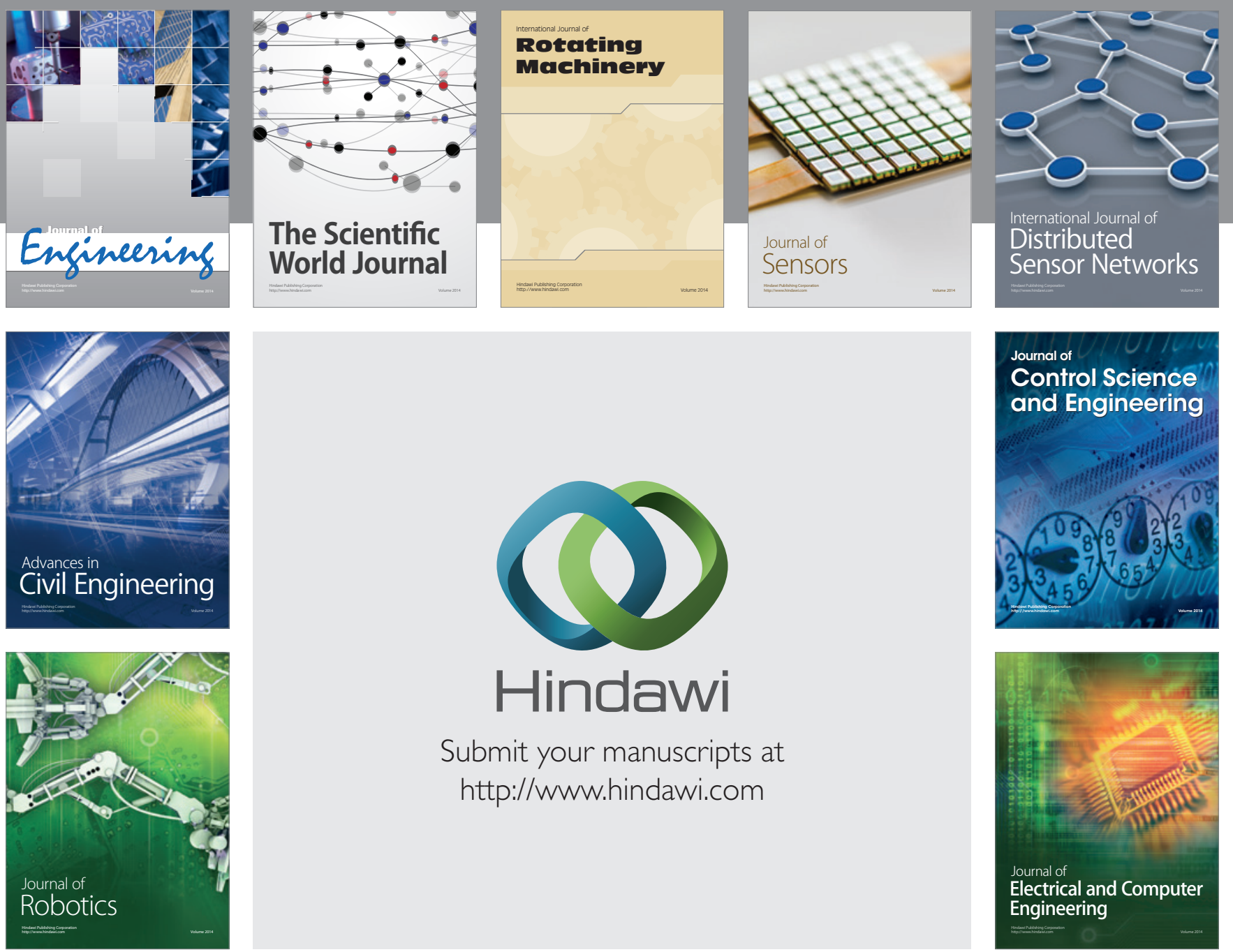

Submit your manuscripts at

http://www.hindawi.com
